Coherency Assessment of the Ecuadorian Power System using Synchrophasor Measurements

\title{
Evaluación de Coherencia en el Sistema Nacional Interconectado Ecuatoriano empleando Mediciones Sincrofasoriales
}

\author{
P.X. Guacán ${ }^{1} \quad$ N.V. Granda ${ }^{1}$ \\ ${ }^{1}$ Escuela Politécnica Nacional, Ecuador \\ E-mail: paul.guacan@epn.edu.ec; nelson.granda@epn.edu.ec
}

Abstract

This paper presents the coherency evaluation of the dynamic coherency in the Ecuadorian power system, using measurements obtained from Synchrophasor Measurement Units (PMUs) located at the main substations of the backbone transmission system.

Based on the time series delivered by the PMUs, such as frequency, rate of change of frequency, voltage or current phasors, data mining techniques are applied to determine the coherent areas. Several batch clustering techniques has been applied to analyze post-contingency signals in an offline environment. For real time analysis, new algorithms based on stream data concepts have been implemented.

For the evaluation of coherent areas using postcontingency data, validation indexes are used to measure the quality of the clustering process, these indexes are calculated based on the density of the data within the same group or the distance between different groups. For the real time coherency analysis, recursive clustering algorithms are studied and implemented. These algorithms are capable of identifying the number of groups, as well as their composition, through the change of its parameters according to the last received information.

Index terms-- Wide Area Measurement System, Synchrophasor Measurement Unit, Dynamic Coherency, Data Mining

\section{Resumen}

Este documento presenta la evaluación de la coherencia dinámica en el Sistema Nacional Interconectado Ecuatoriano (SNI) empleando mediciones obtenidas por Unidades de Medición Sincrofasorial (PMUs) ubicadas en las principales subestaciones del SNI.

A partir de las series de tiempo de frecuencia, tasa de cambio de la frecuencia, fasores de voltaje o corriente entregadas por las PMU, se aplican técnicas de minería de datos para determinar las áreas coherentes existentes. Varias técnicas de agrupamiento en bloque han sido empleadas para el análisis post-contingencia realizado fuera de línea, y también se han implementado nuevas propuestas para el análisis de información en tiempo real basado en el concepto de flujos de datos.

Para la determinación post-contingencia de las áreas coherentes se emplean índices de validación que permiten medir la calidad de las agrupaciones en función de la densidad de los datos dentro de un mismo grupo o la distancia entre diferentes grupos. En cambio, para la evaluación de coherencia en tiempo real, se analizan e implementan algoritmos recursivos de agrupamiento como herramienta para el análisis. Estos algoritmos son capaces de identificar el número de grupos presentes, así como su composición, mediante la adaptación de sus parámetros acorde con la última información recibida.

Palabras clave-- Sistemas de Medición de Área Extendida, Unidades de Medición Sincrofasorial, Coherencia dinámica, Minería de datos. 


\section{INTRODUCCIÓN}

El desarrollo e implementación de los Sistemas de Medición de Área Extendida (WAMS - Wide Area Measurement Systems), basados en Unidades de Medición Sincrofasorial (PMU, por sus siglas en ingles), junto a sistemas de comunicación mejorados ha permitido el monitoreo en tiempo real de los Sistemas Eléctricos de Potencia (SEP) con alta velocidad y excelente precisión [1]. En este sentido, actualmente es posible medir el comportamiento dinámico del sistema y se hace necesario desarrollar aplicaciones que, haciendo uso de la información entregada por los WAMS, permitan determinar acciones de control para mejorar la operación del SEP.

Un fenómeno de relevante importancia en la operación del sistema eléctrico es la coherencia dinámica entre generadores, la cual consiste en la formación de grupos de generadores que frente a una determinada perturbación oscilan de forma coordinada [2]. La identificación de estos grupos coherentes puede ser abordada desde varias perspectivas, siendo dos las más reconocidas: modelos del sistema y mediciones del comportamiento real de los generadores frente a un evento.

Previo al desarrollo de los sistemas avanzados de medición, las metodologías basadas en modelos del sistema fueron las más estudiadas; en los últimos años las metodologías basadas en mediciones han tenido un desarrollo importante.

Sin embargo, la gran cantidad de información entregada por los WAMS hace necesario desarrollar nuevas herramientas que permitan obtener información crítica, conocimiento o patrones que sean relevantes para la operación y control del sistema [3]. Dicho esto, se plantea desarrollar una metodología basada en técnicas de minería de datos que determine los patrones de coherencia que aparecen ante diferentes perturbaciones y que son registrados mediante las PMUs instaladas en el SNI.

Es así como, en la sección 2 se realiza una descripción teórica de la coherencia dinámica y las técnicas de identificación de grupos coherentes mediante algoritmos de agrupamiento. Posteriormente, en la sección 3 se presenta la metodología propuesta para la identificación y evaluación de áreas coherentes empleando técnicas recursivas y de agrupamiento en bloque. En la sección 4, los eventos suscitados en el SNI el 01 de julio de 2016 y el 02 de marzo de 2018, registrados a través del sistema WAMS, son analizados y empleados para realizar la evaluación de coherencia. Finalmente, en la sección 5 se presentan las conclusiones y recomendaciones de este trabajo.

\section{MARCO TEÓRICO}

\subsection{Coherencia Eléctrica}

Un grupo de generadores coherentes, frente a una determinada perturbación, está formando por aquellos generadores que oscilan con la misma velocidad angular $(\omega)$ y que mantienen una relación compleja constante entre sus voltajes en terminales $(E)$ a lo largo de un intervalo de tiempo $(t)$ [2].

$$
\begin{gathered}
\frac{\overline{E_{t}}(t)}{\overline{E_{j}}(t)}=\frac{E_{i}(t)}{E_{j}(t)} e^{j\left[\delta_{i}(t)-\delta_{j}(t)\right]}=k t e \\
\omega_{i}(t)=\omega_{j}(t)
\end{gathered}
$$

Generalmente se dice que dos generadores " $i$ " y “ $j$ " son coherentes cuando al producirse una perturbación éstos oscilan al unísono, es decir, su diferencia angular se mantiene constante dentro de un intervalo de tiempo [4].

$$
\Delta \boldsymbol{\delta}_{i}(\boldsymbol{t})-\Delta \boldsymbol{\delta}_{j}(\boldsymbol{t}) \leq \varepsilon
$$

El concepto de coherencia dinámica entre generadores puede extenderse a las barras del sistema, indicando que un grupo de barras que mantienen una relación compleja constante entre sus voltajes $(V)$ a lo largo de un intervalo de tiempo $(t)$ forman un área coherente. Es decir, las barras dentro de un área coherente mantienen una diferencia angular constante en el intervalo de tiempo. Estas condiciones se expresan como:

$$
\begin{gathered}
\frac{\overline{V_{l}}(t)}{\overline{V_{j}}(t)}=\frac{V_{i}(t)}{V_{j}(t)} e^{j\left[\theta_{i}(t)-\theta_{j}(t)\right]}=k t e \\
\Delta \theta_{i}(t)-\Delta \theta_{j}(t) \leq \varepsilon
\end{gathered}
$$

En este sentido, la identificación de áreas coherentes es un problema que puede ser abordado desde diferentes perspectivas debido a que el agrupamiento de éstas varía con la topología del sistema y las condiciones operativas. Con el incremento en la cantidad de mediciones sincrofasoriales disponibles en los sistemas eléctricos, la evaluación de coherencia en tiempo real se ha vuelto factible y cada vez más estudiada [1].

\subsection{Métodos basados en Mediciones del Sistema}

\subsubsection{Análisis Prony}

El objetivo de este método es aproximar un conjunto de mediciones a través de una suma de términos exponenciales complejos amortiguados dados por (6).

$$
y(t) \approx \widehat{y}(t)=\sum_{i=1}^{n} R_{i} e^{\lambda_{i} t}
$$

Donde: $\boldsymbol{R}_{\boldsymbol{i}}$ es el residuo de salida para el polo de tiempo continuo $\boldsymbol{\lambda}_{\boldsymbol{i}}$ y $\mathrm{n}$ es el orden del modelo [5].

Una vez determinados los modos oscilatorios del sistema, la identificación de áreas coherentes puede ser realizada mediante el agrupamiento de los fasores correspondientes tanto a los modos electromecánicos inter-área $(0.7 \mathrm{a} 1 \mathrm{~Hz})$ como de los modos oscilatorios 
locales $(0.7$ a $1.2 \mathrm{~Hz})$, lo cual es debido a que en este trabajo se considera la información obtenida en las barras del SNI mas no directamente de los generadores del mismo.

\subsubsection{Técnicas de agrupamiento - Clustering}

El proceso de agrupamiento o "Clustering" consiste en agrupar conjuntos de datos acorde a su similitud. Por lo general, este proceso se lo realiza cuando no se dispone de información sobre la pertenencia de los datos a clases predefinidas y por lo tanto se lo clasifica como aprendizaje no supervisado.

El objetivo de este tipo de agrupamiento es que los puntos de un mismo grupo sean "similares" entre sí y "disimilares" a puntos de otros grupos. El concepto de similaridad comúnmente se relaciona con qué tan cercanos se encuentran dos puntos entre sí respecto a una función de distancia [3].

\subsection{Reducción de dimensiones: Análisis de Componentes Principales -PCA}

Debido a su alta velocidad de muestreo, las PMUs entregan grandes cantidades de información a los sistemas WAMS, por lo que en determinadas aplicaciones se requiere reducir el tamaño de las bases de datos. El principal objetivo de realizar una reducción de dimensiones de una matriz de datos es extraer la información más importante y comprimir el tamaño del conjunto de datos conservando la mayor parte de información [6].

El Análisis de Componentes Principales (PCA Principal Component Analysis) es una de técnica de reducción de dimensionalidad muy empleada debido a su eficiencia y simplicidad. Su objetivo es reducir las dimensiones de una matriz de datos $\mathbf{X}$, con $\mathrm{n}$ mediciones $\mathrm{y}$ p dimensiones, desde sus $\mathrm{p}$ dimensiones originales a una matriz de pesos $\mathbf{W}$ con $n$ mediciones $y \quad q$ componentes principales, donde $\mathrm{q} \ll \mathrm{p}[6]$.

\section{METODOLOGÍA PROPUESTA}

La metodología desarrollada inicia con la carga de información desde una base de datos preprocesados [7], continúa con la selección de las variables/señales a analizar y la posterior aplicación de técnicas de minería de datos para la determinación de áreas coherentes, tanto de manera offline como online, y finalmente, la validación de dichas agrupaciones. En la Figura 1 se muestra el diagrama de flujo detallado de todo el proceso para agrupamiento y validación de áreas coherentes.

\subsection{Carga y Selección de Señales}

La base de datos está formada por mediciones obtenidas del sistema WAMS y dado que el objetivo es determinar las áreas coherentes, las señales seleccionadas

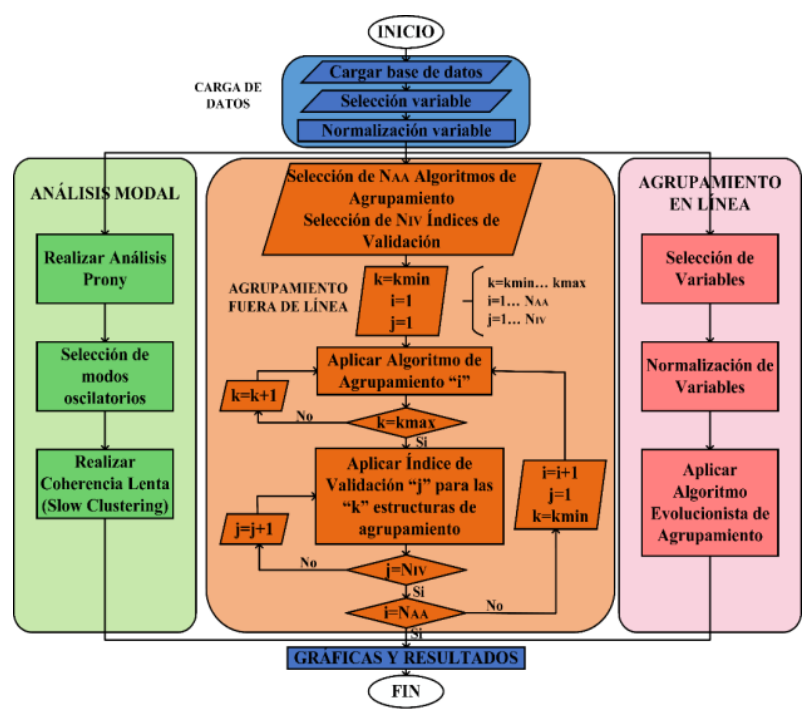

Figura 1: Diagrama de flujo identificación de áreas coherentes

son las mediciones de frecuencia, tasa de cambio de la frecuencia y ángulo del fasor de voltaje de cada barra. Debido a que las señales se miden en unidades diferentes (hertz, radianes) y que pueden existir grandes diferencias entre sus magnitudes es necesario realizar una normalización de las mediciones, para lo cual se emplean 4 métodos diferentes: Z-score, Norma, Escalar, Rango, descritos en la referencia [8].

\subsection{Análisis Modal}

En esta etapa se determina la coherencia entre barras empleando las mediciones disponibles y el método de análisis Prony, el cual ha demostrado ser un método efectivo para determinar los modos oscilatorios electromecánicos en el SNI a partir de sus mediciones sincrofasoriales [9]. Para este fin se emplea la herramienta BPA/PNNL Prony Ringdown [10].

\subsection{Agrupamiento Fuera de Línea}

El proceso consiste en la aplicación de i algoritmos de agrupamiento a la matriz de mediciones PMU con la finalidad de obtener k estructuras de agrupación por cada algoritmo, las estructuras obtenidas son evaluadas mediante $\mathrm{j}$ índices de validación, obteniéndose de esta manera el número óptimo de grupos para cada índice.

\subsubsection{Algoritmos de agrupamiento}

Los algoritmos de agrupamiento en bloque empleados se clasifican en tres categorías: métodos de particionamiento, métodos jerárquicos aglomerativos y métodos basados en funciones de densidad.

- Métodos de particionamiento: Estos algoritmos estiman de manera inicial los centros de los grupos y los elementos más cercanos a éstos son asignados. Una vez se asignan todos los elementos a un grupo, se actualiza el centro de cada grupo y 
se repite el procedimiento hasta que no existan variaciones significativas en las agrupaciones.

Son métodos sencillos y eficaces que tienen la desventaja de requerir el número de grupos que existen en la matriz de datos. En el presente trabajo se emplean los siguientes algoritmos: Kmeans, K-medoids, Partitioning Around Medoids (PAM), Fuzzy C-means (FCM) y Affinity Propagation (AP) [8].

- Métodos Jerárquicos Aglomerativos: Son algoritmos que realizan la agrupación iterativa de cada elemento, los cuales empiezan formando su propio grupo y luego se combinan con el siguiente grupo más cercano hasta unir todos los elementos en un grupo único o alcanzar un criterio de parada. Estos métodos de agrupamiento se diferencian entre sí por la forma en la que se calcula la distancia entre grupos; en el presente trabajo se emplean los siguientes algoritmos: Hierarchical Single (HS), Hierarchical Complete (HC) y Hierarchical Average (HA) [8].

- Métodos basados en funciones de densidad: Son algoritmos diseñados para expandir el tamaño de un grupo cuando la densidad (número de objetos) en la vecindad supere un valor límite. Se han empleado los siguientes algoritmos: Substractive Clustering (SC) y DBSCAN [8].

Se implementó también un esquema de agrupamiento basado en agrupamiento substractivo (SC) aplicado sobre la matriz $\mathbf{W}$ de pesos obtenida del análisis de componentes principales (PCA).

\subsubsection{Validación de agrupamiento mediante Índices}

La validación es una de las cuestiones más importantes para la identificación exitosa de grupos. Como se mencionó previamente, el proceso de agrupamiento es un tipo de aprendizaje no supervisado, por lo cual, no es posible conocer de antemano y con total certeza el número de grupos.

La validación consiste en cuantificar las características de concentración, separación, homogeneidad, etc. entre cada elemento de un grupo o entre diferentes grupos para tener una idea de la calidad de las agrupaciones obtenidas. Con tal objetivo, los índices de validación empleados son: Índice Silhouette (SI), índice Davies-Bouldin (DB), índice CalinskiHarabasz (CH), índice Dunn (D), índice R-Squared (RS), índice Xie-Beni (XB), índice Partition $(\mathrm{P})$ e índice Separation (Sep) [8].

\subsection{Agrupamiento en línea}

En esta etapa se realiza la implementación de dos algoritmos evolucionistas de agrupamiento y uno de ventana móvil, con la finalidad de superar la necesidad de especificar como dato de entrada el número de grupos y posibilitar el trabajo con flujos de datos en tiempo real de las señales registradas mediante las PMU.

El proceso inicia con la formación de la matriz de datos $\mathbf{X}$ mediante la selección de mediciones que describan el comportamiento dinámico del SEP a lo largo del tiempo. Las señales empleadas son: frecuencia, tasa de cambio de la frecuencia y el ángulo del fasor de voltaje. Al tratar con diferentes variables en un mismo instante de tiempo es preciso normalizar estas mediciones, con el fin de que sean comparables entre sí.

$$
X=\left[\begin{array}{cccc}
\boldsymbol{\theta}_{G 1} & \boldsymbol{f}_{G 1} & \ldots & \boldsymbol{d} \boldsymbol{f}_{G 1} \\
\boldsymbol{\theta}_{G 2} & \boldsymbol{f}_{G 2} & \ldots & \boldsymbol{d} \boldsymbol{f}_{G 2} \\
\vdots & \vdots & \ddots & \vdots \\
\boldsymbol{\theta}_{G n} & \boldsymbol{f}_{G n} & \ldots & \boldsymbol{d} \boldsymbol{f}_{G n}
\end{array}\right]_{t=t k}
$$

Posterior a la normalización de las señales se procede a construir de manera recursiva la matriz de similaridad $\mathbf{S}$ con la finalidad de reducir el tiempo de cálculo requerido por el algoritmo para realizar el agrupamiento, característica esencial en aplicaciones usadas en ambientes de tiempo real [8]:

El valor $\boldsymbol{X}_{\boldsymbol{i} j}(\boldsymbol{t})$ representa la diferencia entre las mediciones de dos barras i y j en el instante $t$.

$$
X_{i j}(t)=X_{i}(t)-X_{j}(t)
$$

De manera recursiva se calcula la integral del cuadrado de $\boldsymbol{X}_{\boldsymbol{i} \boldsymbol{j}}(\boldsymbol{t})$ en un intervalo de tiempo $\boldsymbol{T}$, aplicando la regla de integración trapezoidal (9):

$$
Y_{i j}\left(t_{k}\right)=Y_{i j}\left(t_{k-1}\right)+\frac{X_{i j}^{2}\left(t_{k}\right)+X_{i j}^{2}\left(t_{k-1}\right)}{2} \Delta t
$$

Se calcula el índice de coherencia $\boldsymbol{c}_{\boldsymbol{i} j}$ mediante (10)

$$
c_{i j}=\frac{\sqrt{Y_{i j}(t)}}{T}
$$

A partir de los índices $\boldsymbol{c}_{\boldsymbol{i} j}$ se forma la matriz de coherencia $\mathbf{C}$ :

$$
C=\left[\begin{array}{cccc}
c_{11} & c_{12} & \ldots & c_{1 n} \\
c_{21} & c_{22} & \ldots & c_{2 n} \\
\vdots & \vdots & \ddots & \vdots \\
c_{n 1} & c_{n 2} & \ldots & c_{n n}
\end{array}\right]
$$

Finalmente, la matriz de similaridad $\mathbf{S}$ se obtiene mediante (12)

$$
S=I-\frac{C}{\max (C)}
$$

Donde I es una matriz cuadrada llena de unos.

Los algoritmos evolucionistas implementados son Evolving Clustering Method (eCM) [8] y Evolving Takagi Sugeno Clustering (eTS) [8]. El algoritmo de ventana móvil será la adaptación del algoritmo de 
agrupamiento substractivo (SC) en conjunto con el análisis de componentes principales PCA. Para el algoritmo PCA+SC es necesario definir el ancho de la ventana móvil de análisis, para lo cual se emplea la metodología propuesta en [11], que consiste en emplear dos veces el periodo de la frecuencia del modo electromecánico de oscilación más lento determinado mediante análisis Prony.

\section{APLICACIÓN AL SISTEMA NACIONAL INTERCONECTADO}

A partir de las mediciones obtenidas por las PMUs instaladas en el SNI se realizó la evaluación de los grupos coherentes ante diferentes fallas registradas en el sistema. Cabe indicar, que el análisis permite encontrar "áreas coherentes", por cuanto NO se cuenta con mediciones sincrofasoriales en bornes de los generadores del sistema.

\subsection{Caso 1: 01 de julio de 2016}

El 01 de julio de 2016 alrededor de las 10h30 se produjo una falla en el reactor del circuito 1 de la línea de transmisión El Inga - San Rafael de 500 kV ocasionando la apertura de la misma y por ende la salida de la central Coca Codo Sinclair. Los eventos producidos provocaron la desconexión de la interconexión entre Colombia - Ecuador de $230 \mathrm{kV}$ debido a la actuación del Esquema de Separación de Áreas, y la posterior desconexión de carga debido a la activación del Esquema de Alivio de Carga por baja frecuencia. Las principales centrales que se encontraban abasteciendo al SNI previo a la contingencia son: Paute Integral, Coca Codo Sinclair y Marcel Laniado.

\subsubsection{Análisis Prony}

Se evalúan las señales de frecuencia, tasa de cambio de la frecuencia y ángulo del voltaje para determinar los modos electromecánicos (Ver Tabla 1), acorde al rango de frecuencia de estos, dando preferencia a aquellos con la mayor amplitud.

Tabla 1: Resultados de BPA/PNNL Ringdown Analysis Toolbox

\begin{tabular}{|c|c|c|c|}
\hline Variable & $\begin{array}{c}\text { Frecuencia del modo } \\
\text { oscilatorio }[\mathrm{Hz}]\end{array}$ & Amplitud & $\begin{array}{c}\text { Nro. grupos } \\
\text { determinados }\end{array}$ \\
\hline $\boldsymbol{f}$ & 0.802 & 1028.18 & 3 \\
\hline $\boldsymbol{d} \boldsymbol{f} / \boldsymbol{d} \boldsymbol{t}$ & 1.196 & 0.6773 & 3 \\
\hline $\boldsymbol{\theta}_{\boldsymbol{v}}$ & 1.01 & 400 & 3 \\
\hline
\end{tabular}

El intervalo de análisis es de 7s, tiempo suficiente para caracterizar la coherencia del sistema. En [12] se presentan una caracterización estadística de los modos oscilatorios esperados en el SNI, siendo algunas de ellas 0.6, 0.7 y $1.2 \mathrm{~Hz}$, validando de esta manera las frecuencias determinadas mediante la metodología presentada (Ver Tabla 1).

Mediante este método se determinó, empleando las señales de frecuencia y tasa de cambio de frecuencia, la existencia de tres áreas coherentes (Ver Tabla 2). Sin embargo, la señal del ángulo del voltaje no presenta el mismo patrón de coherencia del sistema y se descarta en los análisis posteriores.

En la Figura 2 se presenta los fasores correspondientes al modo oscilatorio $\boldsymbol{f}=\mathbf{1}, 196 \mathrm{~Hz}$ de la tasa de cambio de la frecuencia $(d f / d t)$ y en la Figura 3 se indican las agrupaciones realizadas, las cuales son las mismas para la señal de frecuencia.

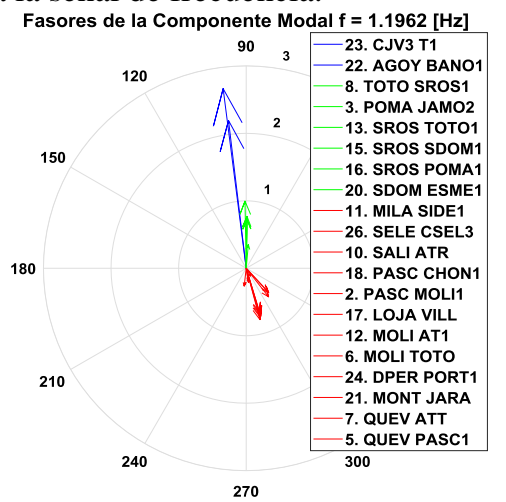

Figura 2: Fasores de la componente modal, derivada de la frecuencia

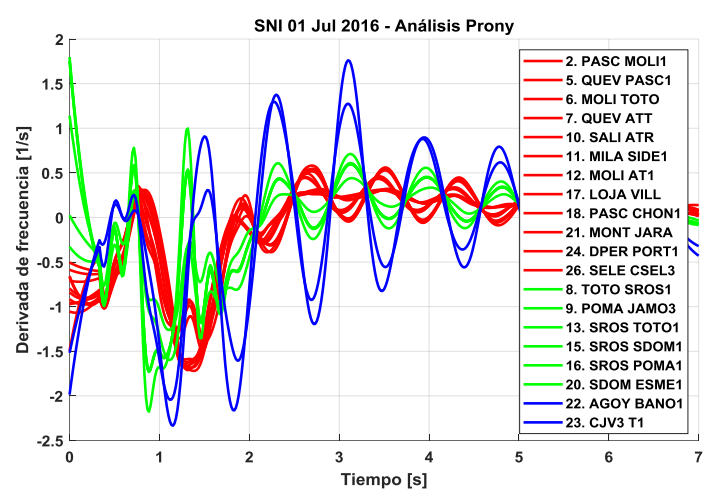

Figura 3: Agrupamiento mediante análisis Prony de la tasa de cambio de la frecuencia

Tabla 2: Resultados Análisis Prony - Caso 01 de julio 2016

\begin{tabular}{|c|c|c|c|}
\hline Área & Subestación & Posición & Voltaje \\
\hline \multirow{15}{*}{1} & C. Trinitaria & TV1 & $13.8 \mathrm{kV}$ \\
\hline & D. Peripa & Portoviejo 1 & $138 \mathrm{kV}$ \\
\hline & Loja & Villonaco & $69 \mathrm{kV}$ \\
\hline & Milagro & San Idelfonso 1 & $138 \mathrm{kV}$ \\
\hline & \multirow{4}{*}{ Molino } & AT1 & $138 \mathrm{kV}$ \\
\hline & & Taday & $230 \mathrm{kV}$ \\
\hline & & Pascuales1 & $230 \mathrm{kV}$ \\
\hline & & Totoras & $230 \mathrm{kV}$ \\
\hline & \multirow{3}{*}{ Pascuales } & Chone 1 & $138 \mathrm{kV}$ \\
\hline & & Molino 1 & $230 \mathrm{kV}$ \\
\hline & & Molino 2 & $230 \mathrm{kV}$ \\
\hline & \multirow{2}{*}{ Quevedo } & ATT & $138 \mathrm{kV}$ \\
\hline & & Pascuales 1 & $230 \mathrm{kV}$ \\
\hline & S. Elena & C. S. Elena III & $69 \mathrm{kV}$ \\
\hline & Salitral & ATR & $138 \mathrm{kV}$ \\
\hline \multirow{6}{*}{2} & \multirow{2}{*}{ Pomasqui } & Jamondino 2 & $230 \mathrm{kV}$ \\
\hline & & Jamondino 3 & $230 \mathrm{kV}$ \\
\hline & \multirow{4}{*}{ Santa Rosa } & Pomasqui 1 & $230 \mathrm{kV}$ \\
\hline & & Santo Domingo 1 & $230 \mathrm{kV}$ \\
\hline & & Totoras 1 & $230 \mathrm{kV}$ \\
\hline & & Totoras 2 & $230 \mathrm{kV}$ \\
\hline
\end{tabular}




\begin{tabular}{|c|c|c|c|}
\hline \multirow{2}{*}{} & \multirow{2}{*}{ Sto. Domingo } & Baba & $230 \mathrm{kV}$ \\
\cline { 2 - 4 } & Totoras & Esmeraldas & $138 \mathrm{kV}$ \\
\hline \multirow{2}{*}{3} & Santa Rosa 1 & $230 \mathrm{kV}$ \\
\cline { 2 - 4 } & C. Jivinán III & Baños 1 & $138 \mathrm{kV}$ \\
\hline
\end{tabular}

\subsubsection{Agrupamiento fuera de línea}

Al aplicar los algoritmos de agrupamiento y los índices de validación mencionados en la sección 3.3, se determinó que, considerando las variables frecuencia y tasa de cambio de la frecuencia, existen tres áreas coherentes. En la Figura 4 se presentan los resultados de los índices de validación aplicados.

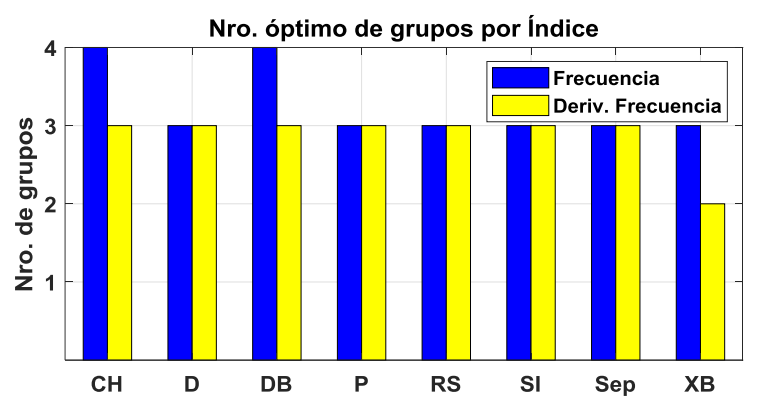

Figura 4: Resultados de los índices de validación, 01-07-2016

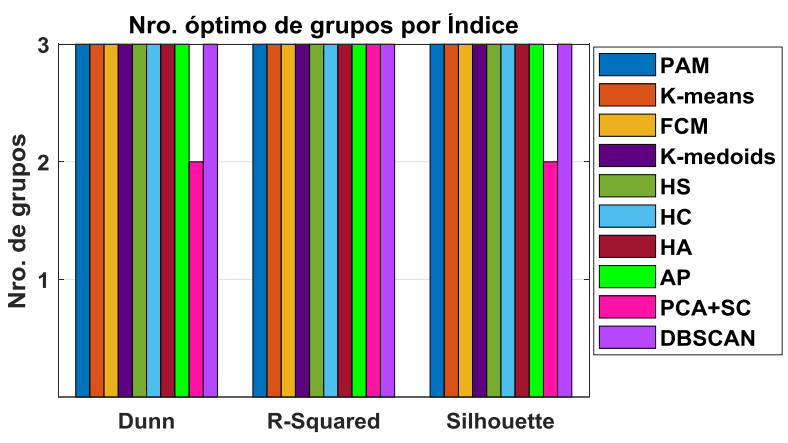

Figura 5: Número óptimo de grupos, frecuencia

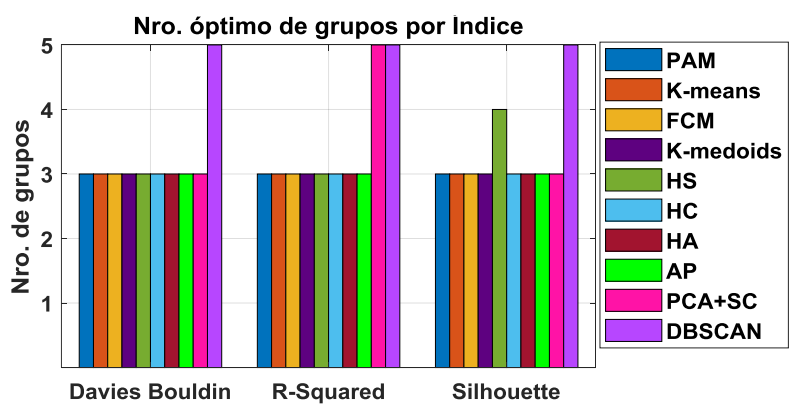

Figura 6: Número óptimo de grupos, derivada de la frecuencia

En la Figura 5 y Figura 6 se detallan los conjuntos: algoritmo de agrupamiento - índice de validación que obtuvieron los mejores resultados. Se destacan los algoritmos que emplean métodos de particionamiento y métodos jerárquicos, considerando el número óptimo de grupos y la composición de las agrupaciones, siendo idénticas a las obtenidas mediante análisis Prony (Tabla
2). Los algoritmos basados en funciones de densidad fueron poco efectivos debido a la alta variabilidad de los datos para la falla suscitada.

\subsubsection{Agrupamiento en línea}

Se consideraron las variables de frecuencia y tasa de cambio de la frecuencia para la elaboración de la matriz de datos $\mathbf{X}$ que emplea cada algoritmo.

- Evolving Clustering Method (eCM): Previamente, se normaliza la matriz de datos de entrada X mediante el método Rango, y, con el objetivo de obtener agrupaciones continuas a lo largo del intervalo de tiempo, se calculó recursivamente la matriz de similaridad S en cada instante de tiempo. El algoritmo identificó los mismos grupos coherentes mencionados en la Tabla 2, presentando ciertos errores al inicio del proceso de agrupamiento.

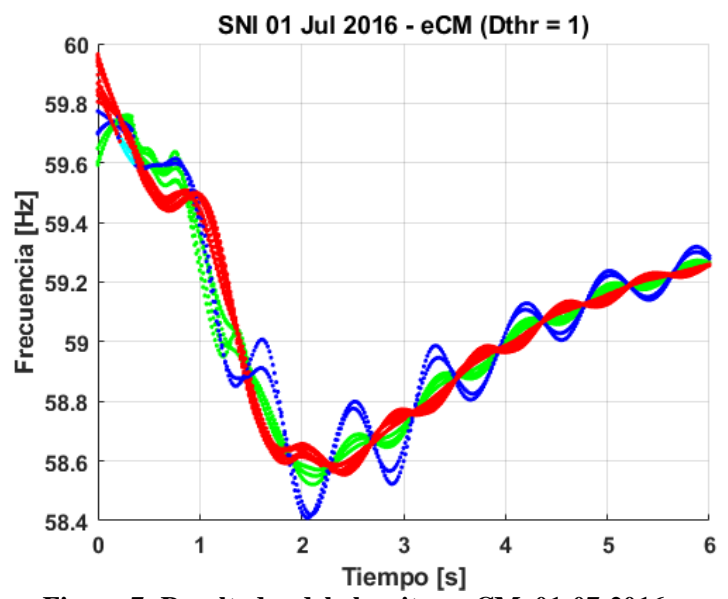

Figura 7: Resultados del algoritmo eCM, 01-07-2016

- Evolving Takagi-Sugeno (eTS): En este caso, se emplean el método Rango para normalizar la matriz de datos $X$ y se calcula la matriz de similaridad S. Inicialmente, el algoritmo identifica solamente 2 grupos, para posteriormente auto-corregirse e identificar correctamente los tres grupos indicados en la Tabla 2, conforme varía la dinámica del sistema.

- PCA+Substractive Clustering: Para este esquema se aplicaron los diferentes métodos de normalización a la matriz X y se calculó la matriz de similaridad $\mathrm{S}$, sin obtener un resultado aceptable que permita caracterizar la coherencia del sistema, considerando un ancho de la ventana móvil de $2.5 \mathrm{~s}$. Los resultados obtenidos son similares a los de la etapa de agrupamiento fuera de línea empleando algoritmos basados en funciones de densidad, lo cual podría explicarse por el hecho de que el algoritmo SC, usa una función de densidad para definir el agrupamiento óptimo. 


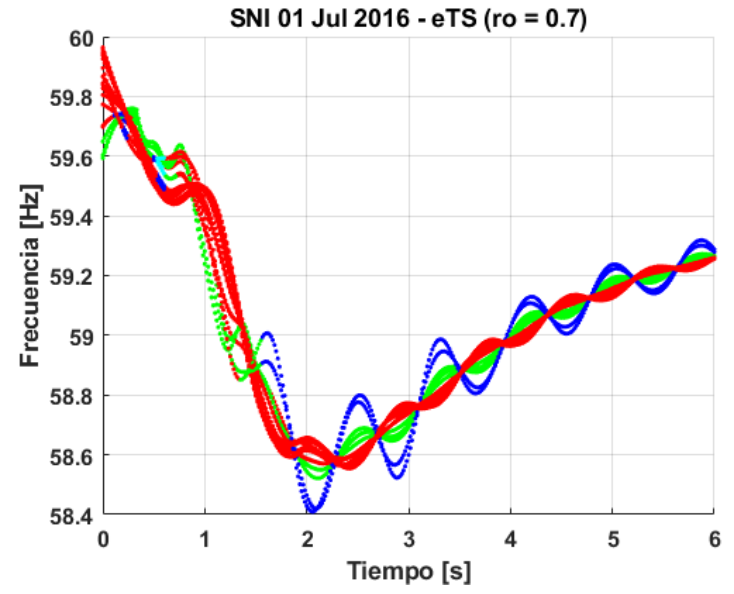

Figura 8: Resultados del algoritmo eTS, 01-07-2016

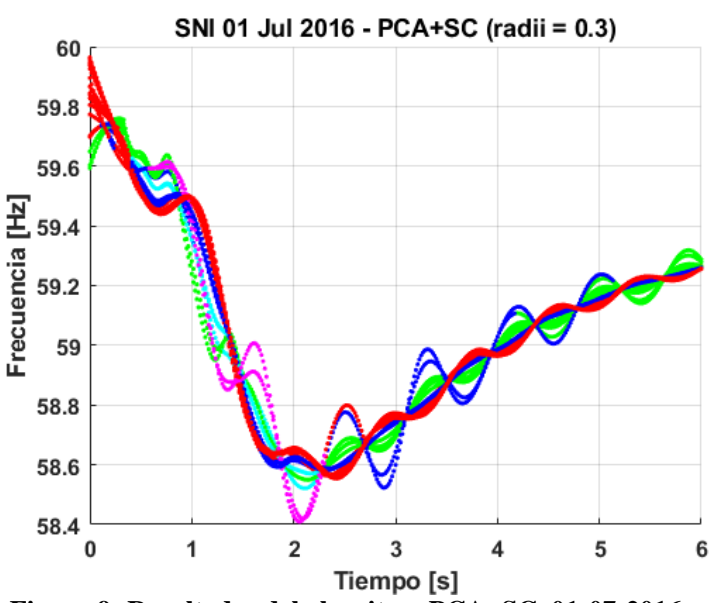

Figura 9: Resultados del algoritmo PCA+SC, 01-07-2016

\subsection{Caso 2: 02 de marzo de 2018}

El 02 de marzo de 2018, a las $15 \mathrm{~h} 21$ se produjo el disparo de la L/T Quevedo - Chorrillos de $230 \mathrm{kV}$, ocasionado la activación del Esquema de Protección Sistémica (SPS), luego de lo cual, cuando ya empieza a estabilizarse el sistema, ocurre el disparo de la L/T Totoras - Molino de $230 \mathrm{kV}$ ocasionando la salida en cascada de elementos de generación y transmisión con la consiguiente separación del SNI en dos islas independientes. Las principales subestaciones conectadas al SNI previo a la contingencia son: Paute Integral, Coca Codo, Termoesmeraldas, Machala, Pomasqui, etc.

\subsubsection{Análisis Prony}

Para el caso de estudio actual se evaluaron las señales de frecuencia y ángulo del voltaje, señales de las cuales se determinan los modos electromecánicos (Tabla 3). El tiempo de análisis es de 10s. De igual manera, en [12] se presentan las frecuencias características de 0.2 y $0.7 \mathrm{~Hz}$, las cuales son muy similares a las determinadas mediante análisis Prony, por lo cual respaldan estos resultados.
Tabla 3: Resultados de BPA/PNNL Ringdown Analysis Toolbox

\begin{tabular}{|c|c|c|c|}
\hline Variable & $\begin{array}{c}\text { Frecuencia del modo } \\
\text { oscilatorio [Hz] }\end{array}$ & Amplitud & $\begin{array}{c}\text { Nro. grupos } \\
\text { determinados }\end{array}$ \\
\hline $\boldsymbol{f}$ & 0.701 & 795340 & 2 \\
\hline $\boldsymbol{\theta}_{v}$ & 0.222 & 0.4497 & 2 \\
\hline
\end{tabular}

Se determinó, tanto para la señal de frecuencia como para el ángulo del voltaje, la existencia de dos áreas coherentes (Tabla 4).

Tabla 4: Resultados del Análisis Prony - Caso 02 de marzo 2018

\begin{tabular}{|c|c|c|c|}
\hline Grupo & Subestación & Posición & Voltaje \\
\hline \multirow{4}{*}{1} & Zhoray & Milagro 2 & $230 \mathrm{kV}$ \\
\hline & Machala & San Idelfonso 1 & $138 \mathrm{kV}$ \\
\hline & S. Elena & C. S. Elena III & $69 \mathrm{kV}$ \\
\hline & Loja & Villonaco & $69 \mathrm{kV}$ \\
\hline \multirow{20}{*}{2} & \multirow{3}{*}{ Coca Codo } & $\begin{array}{c}\text { San Rafael } 1 \\
\end{array}$ & $500 \mathrm{kV}$ \\
\hline & & San Rafael 2 & $500 \mathrm{kV}$ \\
\hline & & U1 & $500 \mathrm{kV}$ \\
\hline & Due & San Rafael & $230 \mathrm{kV}$ \\
\hline & \multirow{2}{*}{ Pomasqui } & Jamondino 2 & $230 \mathrm{kV}$ \\
\hline & & Jamondino 3 & $230 \mathrm{kV}$ \\
\hline & \multirow{4}{*}{ Santa Rosa } & Pomasqui 1 & $230 \mathrm{kV}$ \\
\hline & & Santo Domingo 1 & $230 \mathrm{kV}$ \\
\hline & & Totoras 1 & $230 \mathrm{kV}$ \\
\hline & & Totoras 2 & $230 \mathrm{kV}$ \\
\hline & \multirow{2}{*}{ Sto. Domingo } & Baba & $230 \mathrm{kV}$ \\
\hline & & Esmeraldas & $138 \mathrm{kV}$ \\
\hline & \multirow[t]{2}{*}{ Quevedo } & ATT & $138 \mathrm{kV}$ \\
\hline & & Pascuales 1 & $230 \mathrm{kV}$ \\
\hline & Totoras & Santa Rosa 1 & $230 \mathrm{kV}$ \\
\hline & Agoyán & Baños 1 & $138 \mathrm{kV}$ \\
\hline & C. Pucará & $\mathrm{T} 2$ & $138 \mathrm{kV}$ \\
\hline & D. Peripa & $\begin{array}{l}\text { Portoviejo } 1 \\
\end{array}$ & $138 \mathrm{kV}$ \\
\hline & Montecristi & Jaramijó & $138 \mathrm{kV}$ \\
\hline & C. Esmeraldas & G1 & $13.8 \mathrm{kV}$ \\
\hline
\end{tabular}

En la Figura 10 se presentan los diferentes fasores correspondientes al modo oscilatorio con $f=0,222 \mathrm{~Hz}$, del ángulo del voltaje y en la Figura 11 se indican los grupos coherente obtenidos, los cuales son idénticos para las dos señales analizadas.

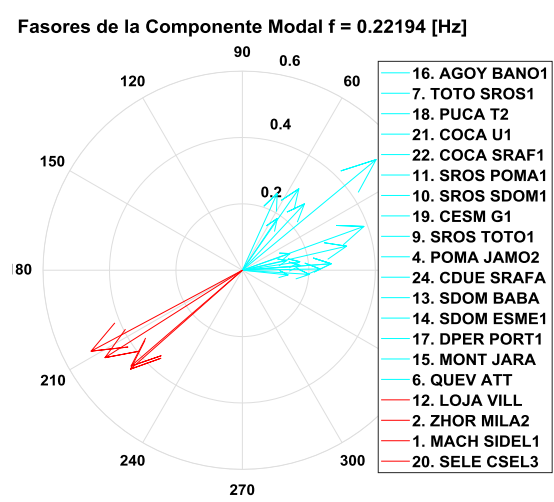

Figura 10: Fasores de la componente modal, ángulo del voltaje 


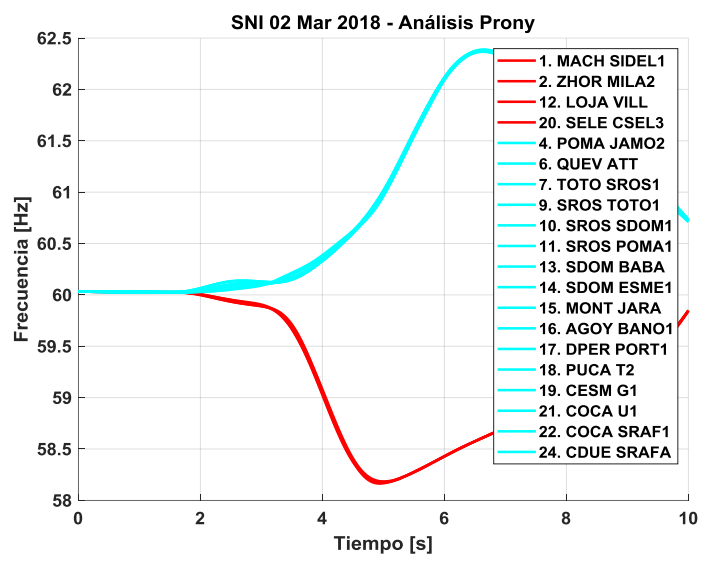

Figura 11: Agrupamiento mediante análisis Prony de la frecuencia

\subsubsection{Agrupamiento fuera de línea}

Los resultados de la metodología empleada determinan que, cuando se analizan las señales de frecuencia, existen dos áreas coherentes, idénticas a las determinadas mediante análisis Prony; mientras que para las señales del ángulo del voltaje se determinaron cuatro grupos. Los dos grupos adicionales corresponden a las mediciones de la PMU Coca U1 y la PMU Zhoray Milagro 2, respectivamente. Este nuevo agrupamiento se debe a que dichas señales difieren en magnitud respecto del resto de las señales de ángulo de voltaje por cortos periodos de tiempo, pudiendo ser la causa un error al momento de la medición. Sin embargo, no ameritan la formación de nuevos grupos independientes.

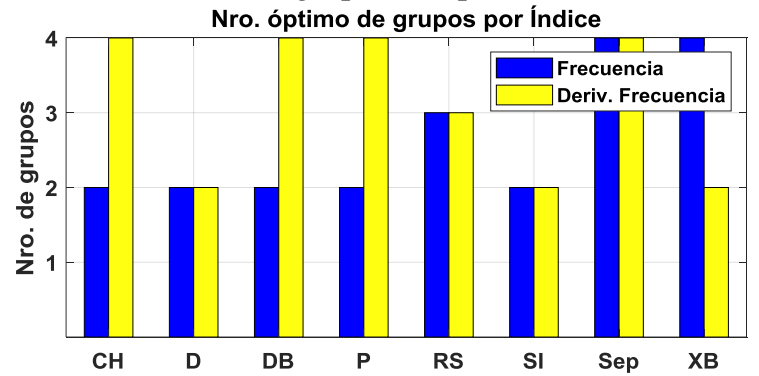

Figura 12: Resultados de los índices de validación, 02-03-2016

En la Figura 13 se presenta la señal del ángulo del voltaje, en la cual se han identificado cuatro grupos, y se hace énfasis en que tanto las mediciones de la PMU Coca U1 y la PMU Zhoray - Milagro 2 pueden unirse perfectamente con uno de los otros grupos.

En la Figura 14 y Figura 15 se presentan con mayor detalle los conjuntos algoritmo de agrupamiento - índice de validación que obtuvieron como resultado dos grupos, según lo indicado en la Tabla 4.

Para este caso en particular, en el que las señales se encuentran claramente diferenciadas entre sí, se logra fácilmente una correcta identificación de las áreas coherentes del sistema utilizando algoritmos de agrupamiento.

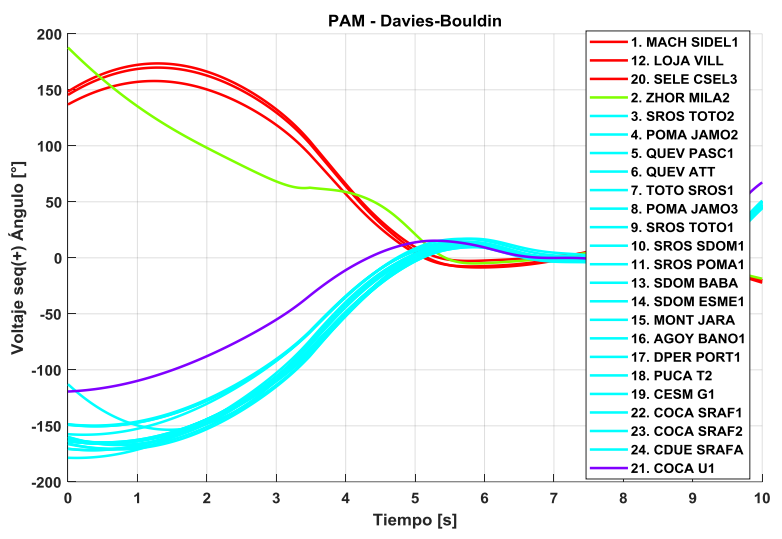

Figura 13: Agrupaciones del ángulo de voltaje

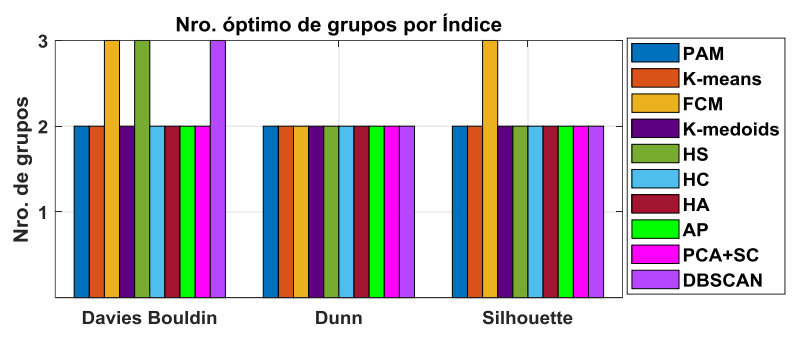

Figura 14: Número óptimo de grupos, frecuencia

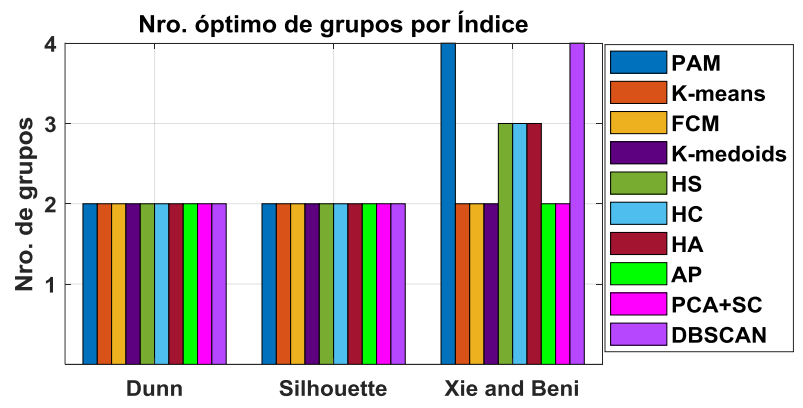

Figura 15: Número óptimo de grupos, ángulo del voltaje

\subsubsection{Agrupamiento Online}

Debido a que no se cuenta con las mediciones de la tasa de cambio de la frecuencia, se emplean solamente las variables de frecuencia y ángulo del fasor de voltaje para la formación de la matriz de datos $\mathbf{X}$. Sin embargo, con la finalidad de mejorar las agrupaciones obtenidas, posteriormente se calcula e incorpora la tasa de cambio de frecuencia a la matriz de entrada.

- Evolving Clustering Method (eCM): Se normaliza la matriz de datos $\mathrm{X}$ mediante el método Z-score, luego de lo cual se calcula la matriz de similaridad $\mathrm{S}$ de forma recursiva a cada instante de tiempo.

En la Figura 16 se observa como el algoritmo eCM determina la existencia de dos áreas coherentes iguales a las indicadas en la Tabla 4, mismas que son consistentes a lo largo del intervalo de tiempo de análisis. 


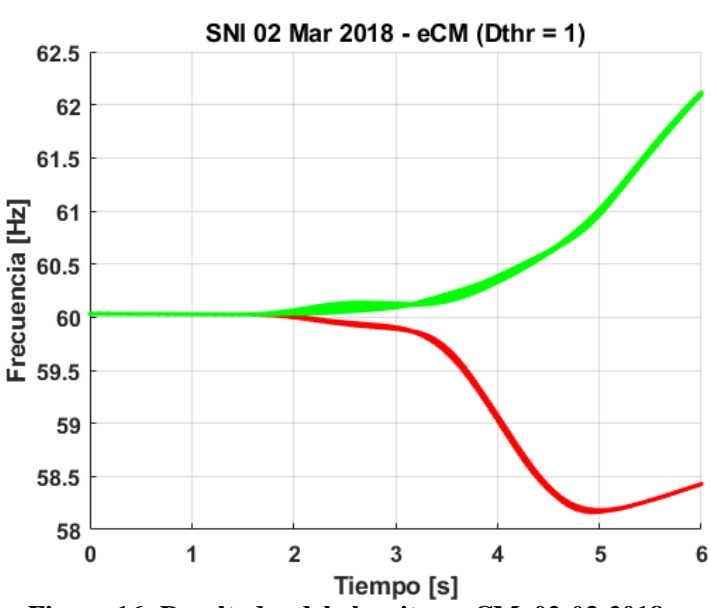

Figura 16: Resultados del algoritmo eCM, 02-03-2018

- Evolving Takagi-Sugeno (eTS): Para emplear este algoritmo fue necesario calcular e incorporar la tasa de cambio de frecuencia a la matriz de datos X, la cual fue normalizada mediante el método Z-score.

En la Figura 17 se observa la identificación de dos grupos (Ver Tabla 4) a lo largo del tiempo de análisis, con ciertos intervalos en los cuales el

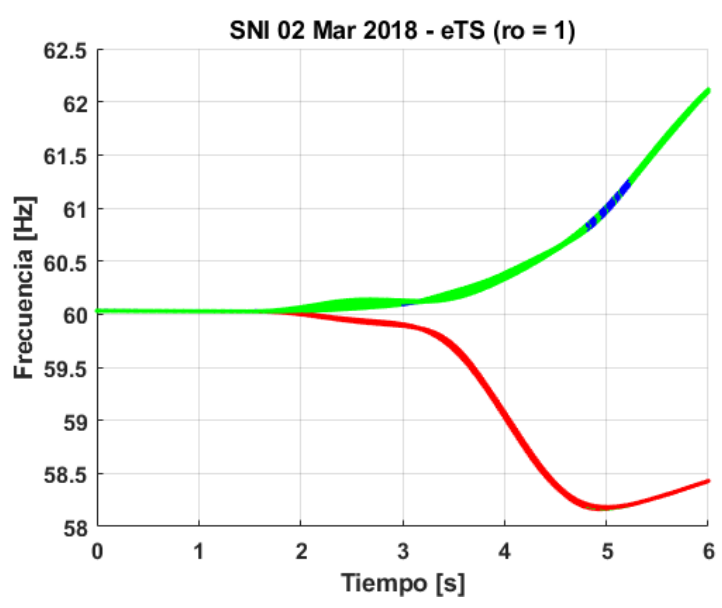

Figura 17: Resultados del algoritmo eTS, 02-03-2018

algoritmo intenta agregar una nueva agrupación (4.82s-5.22s); dicho comportamiento se debe a que eventualmente las mediciones de la PMU Zhoray - Milagro 2 se separan de su grupo y para posteriormente volver a unirse al mismo. Un comportamiento similar se observa con las mediciones de la PMU Coca Codo U1, tal como se explicó en la sección 4.2.2.

- PCA+Substractive Clustering: La matriz de entrada X está formada con las mediciones de frecuencia y ángulo del voltaje, no se aplicó el proceso de normalización, pero se calcula la matriz de similaridad $\mathrm{S}$. Los resultados se muestran en la Figura 18 en los cuales se aprecia claramente la formación de 2 grupos a lo largo del intervalo de tiempo analizado.

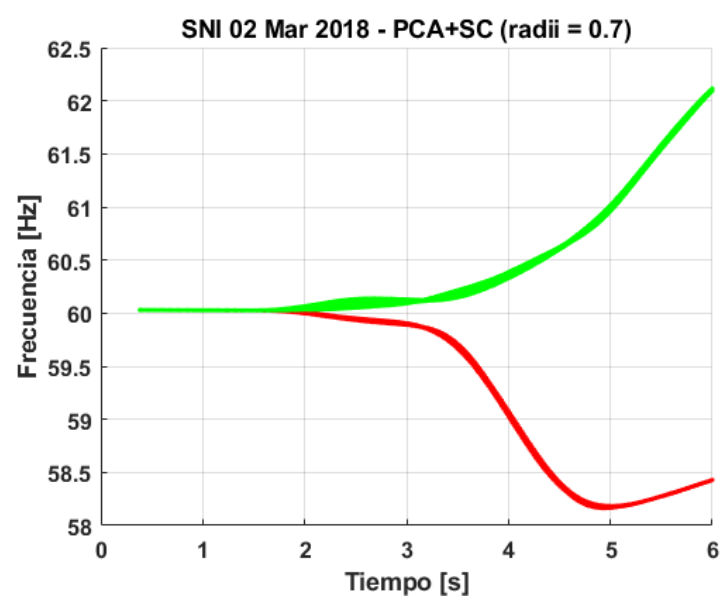

Figura 18: Resultados del algoritmo PCA+SC, 02-03-2018

\section{CONCLUSIONES Y RECOMENDACIONES}

La característica de coherencia eléctrica en un SEP es altamente dependiente del estado operativo del mismo y de las perturbaciones que puedan producirse. Por tal motivo, la metodología planteada para la identificación de áreas coherentes del SNI a partir de las mediciones sincrofasoriales permite obtener resultados que reflejan fielmente dicha característica ante diferentes perturbaciones.

En la etapa de análisis fuera de línea, se probaron varios algoritmos de agrupamiento en bloque, donde los algoritmos: K-means, Affinity Propagation (AP), Hierarchical Complete (HC) y Hierarchical Average (HA) en conjunto con los índices de validación: Silhouette (SI), Dunn (D), Davies - Bouldin (DB) y RSquared (RS) presentan un desempeño sobresaliente, identificando adecuadamente tanto el número óptimo de grupos como la composición de los mismos. En este sentido, para futuros análisis pueden implementarse estos métodos.

Los algoritmos de agrupamiento basados en funciones de densidad no resultaron confiables debido a la alta variabilidad de las señales, estos algoritmos son eficaces cuando los grupos se encuentran claramente definidos, caso poco probable luego de un disturbio en el sistema donde la dinámica del mismo es muy variable.

Las señales de frecuencia y derivada de la frecuencia son las indicadas para caracterizar el patrón de coherencia del sistema, el cual es extraído adecuadamente mediante la aplicación de los algoritmos evolucionistas de agrupamiento implementados: eCM y eTS. Estos algoritmos entregan resultados de buena calidad, son capaces de adaptarse a los cambios del comportamiento dinámico del sistema y realizar exitosamente la evaluación de coherencia en tiempo real. Como medidas adicionales para mejorar las agrupaciones obtenidas se recomienda: la normalización de la matriz de datos de entrada $\mathbf{X}$ de estos algoritmos mediante los 
métodos Z-score y Rango, además del cálculo recursivo de la matriz de similaridad.

Las áreas coherentes obtenidas mediante la metodología planteada pueden ser empleadas en una amplia variedad de aplicaciones relevantes para el análisis y operación del SNI, entre ellas, equivalentes dinámicos del SEP, esquemas de separación de islas, ubicación de eventos dinámicos, cálculo de la frecuencia promedio del SNI, etc. La implementación de esta metodología puede realizarse en cualquier lenguaje de programación para su posterior enlace con el concentrador de datos sincrofasoriales (PDC) que recibe y centraliza las señales de cada PMU del sistema.

\section{REFERENCIAS BIBLIOGRÁFICAS}

[1] J. Cepeda, D. Echeverria, and G. Arguello, "Cenace's experiences on implementing a wide area monitoring system (WAMS) in the Ecuadorian power system," in 2014 IEEE Central America and Panama Convention (CONCAPAN XXXIV), Panama, Panama, 2014, pp. 1-7,

[2] A. J. Germond and R. Podmore, "Dynamic Aggregation of Generating Unit Models," IEEE Trans. Power Appar. Syst., vol. PAS-97, no. 4, pp. 1060-1069, Jul. 1978

[3] J. Han, J. Pei, and M. Kamber, Data Mining: Concepts and Techniques, 3rd ed. Elsevier, 2011.

[4] A. Gallai and R. Thomas, "Coherency identification for large electric power systems," IEEE Trans. Circuits Syst., vol. 29, no. 11, pp. 777-782, Nov. 1982

[5] H. Iswadi, R. Best, and D. Morrow, "Identification of small signal oscillation mode parameters from simulated and actual PMU ringdown data" in 2015 IEEE Eindhoven PowerTech, Eindhoven, Netherlands, 2015, pp. 1-6

[6] H. Abdi and L. J. Williams, "Principal component analysis," Wiley Interdiscip. Rev. Comput. Stat., vol. 2, no. 4, pp. 433-459, Jul. 2010, doi: 10.1002/wics.101.

[7] D. F. Guevara, "Preprocesamiento de los datos de las Unidades de Medición Sincrofasorial (PMUs) utilizando la técnica limpieza de datos - Aplicación al Sistema Nacional Interconectado Ecuatoriano", Escuela Politécnica Nacional, Quito, Ecuador, Jan. 2019

[8] P. X. Guacán, "Evaluación de coherencia entre generadores del SNI empleando mediciones sincrofasoriales", Escuela Politécnica Nacional, Quito, Ecuador, Oct. 2019.

[9] A. Quinaluiza, “Análisis de estabilidad de pequeña señal utilizando mediciones sincrofasoriales
PMU”, Escuela Politécnica Nacional, Quito, Ecuador, Jun. 2014.

[10] "Dynamic System Identification Toolbox | North American SynchroPhasor Initiative." [Online]. Available: https://www.naspi.org/node/490.

[11] N. Granda, "Esquema Adaptable de Separación Controlada en Islas para Sistemas Eléctricos", Ph.D. thesis, Universidad de San Juan, Argentina, Dec. 2015.

[12] R. Cubillo, "Caracterización de la Estabilidad Oscilatoria del Sistema Eléctrico Ecuatoriano mediante la identificación de patrones de oscilación a partir de Mediciones Sincrofasoriales", Ph.D. thesis, Escuela Politécnica Nacional, Quito, Ecuador, Sep. 2018.

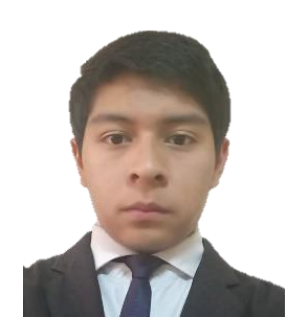

Paul X. Guacán.- Realizó sus estudios secundarios en el Colegio Técnico Salesiano Don Bosco". Obtuvo su título de Bachiller Técnico en Instalaciones, Equipos y Máquinas Eléctricas en julio del 2013. Sus estudios de tercer nivel los realizó en la Escuela Politécnica Nacional. Obtuvo el título de Ingeniero Eléctrico en la en el año 2019.

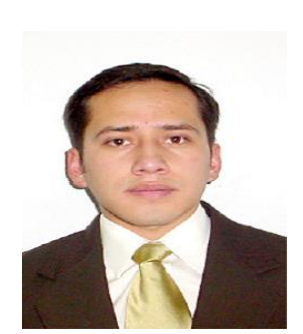

Nelson V. Granda.- Obtuvo el título de Ingeniero Eléctrico en la Escuela Politécnica Nacional en el año 2006 y de Doctor en Ciencias de la Ingeniería Eléctrica en la Universidad Nacional de San Juan (Argentina), en el año 2015. Se ha desempeñado como Ingeniero Eléctrico en varias instituciones del sector eléctrico y petrolero de país. Actualmente se desempeña como parte del staff docente del Departamento de Energía Eléctrica de la Escuela Politécnica Nacional. Sus áreas de interés son análisis y control de sistemas eléctricos de potencia en tiempo real y aplicaciones de Sistemas de Medición de Área extendida (WAMS) basados en unidades de medición sincrofasorial (PMU). 